\title{
Hot Deformation Behavior and Processing Maps of a Medium Manganese TRIP Steel
}

\author{
Ning Yan ${ }^{1} \cdot$ Hong-Shuang $\mathrm{Di}^{1} \cdot$ Hui-Qiang Huang ${ }^{1} \cdot$ R. D. K. Misra ${ }^{2} \cdot$ Yong-Gang Deng ${ }^{1}$
}

Received: 5 September 2018/Revised: 30 October 2018/Published online: 24 November 2018

(C) The Chinese Society for Metals and Springer-Verlag GmbH Germany, part of Springer Nature 2018

\begin{abstract}
The hot deformation behavior of a medium-Mn steel was studied in terms of hot compression flow curves in the temperature range of $850-1050{ }^{\circ} \mathrm{C}$ and strain rates of $0.05-10 \mathrm{~s}^{-1}$. The thermo-mechanical analysis was carried out and suggested that the microstructure during deformation was completely austenite which had high tendency for dynamic recrystallization (DRX). The flow behavior was characterized by significant flow softening at deformation temperatures of 950-1050 ${ }^{\circ} \mathrm{C}$ and lower strain rates of $0.05-5 \mathrm{~s}^{-1}$, which was attributed to heating during deformation, DRX and flow instability. A step-by-step calculating procedure for constitutive equations is proposed. The verification of the modified equations indicated that the developed constitutive models could accurately describe the flow softening behavior of studied steel. Additionally, according to the processing maps and microstructure analysis, it suggested that hot working of mediumMn steel should be carried out at $1050{ }^{\circ} \mathrm{C}$, and the strain rate of $0.05-10 \mathrm{~s}^{-1}$ resulted in significantly recrystallized microstructures in the in steel. The flow localization is mainly flow instability mechanism for experimental steel.
\end{abstract}

Keywords Medium-Mn steel · Hot deformation behavior - Constitutive equations - Flow instability mechanism . Recrystallized microstructure

\section{Introduction}

The primary requirements in the automobile industry are weight reduction, environmental protection and improved safety. These requirements have encouraged the development of new generation of advanced high-strength steels (AHSS). Currently, medium-Mn steels (containing $\sim 3-10 \% \mathrm{Mn})$ as the third generation of AHSS have attracted significant attention due to more reasonable cost compared to TWIP (twinning induced plasticity) steels and their remarkable properties [1-3]. After hot rolling, medium-Mn steels mainly contain $\alpha$-martensite as a

Available online at http://link.springer.com/journal/40195

Hong-Shuang Di

dhshuang@mail.neu.edu.cn

1 State Key Laboratory of Rolling and Automation, Northeastern University, Shenyang 110819, China

2 Laboratory for Excellence in Advanced Steel Research, Department of Metallurgical, Materials and Biomedical Engineering, University of Texas El Paso, El Paso, TX 79968, USA microstructural constituent and transform to significant content of austenite during intercritical annealing, leading to good mechanical properties $[4,5]$. The previous studies on medium-Mn steels mainly focused on the microstructure and mechanical properties $[2,6,7]$, the mechanism of reversal transformation [8,9], the stability of austenite during intercritical annealing [3], the effect of alloying elements $[10,11]$ and carbide precipitation or cementite formed during annealing [12, 13]. However, thermo-mechanical processing (the first step of processing) is complex prior to heat treatment and needs further attention to systematically understand the deformation behavior of medium-Mn steels.

In order to understand the deformation behavior of material during hot forming, it is necessary to develop constitutive equations describing the hot flow behavior based on the deformation mechanisms. A few studies focused on developing constitutive equations to describe the hot flow behavior of stainless steel, magnesium alloy and superalloy [14-20]. Lin and Chen [17] critically reviewed and summarized different constitutive models. The complex deformation mechanism of materials can be reflected in terms of the flow stress, and some aspects are as 
follow: (1) the work hardening (WH) rate is higher than the softening rate induced by dynamic recovery (DRV), resulting in rapidly increasing stress at micro-strain, (2) the stress tends to level off with increased strain because of the competition between work hardening and the softening (DRV), then (3) the dynamic recrystallization (DRX) takes place and the flow stress sharply drops. However, few researches on hot flow behavior of medium steel were reported, which needs further study. Thus, for medium steel, it is necessary to study the deformation mechanism during hot processing.

Moreover, the processing map is an effective supplementary technique to be used for designing and optimizing hot processing parameters. Based on the dynamic materials model (DMM) [21-26], the processing map can predict the relationship between unstable region and deformation condition, thereby providing information on the flow behavior. More importantly, according to the power dissipation maps, the effect of deformation parameters on microstructural evolution can be studied. Therefore, combining with power dissipation model, establishing processing map is beneficial to predict more accurately flow instability and further study the microstructure evolution mechanism.

In this work, the hot flow behavior of medium-Mn steel has been studied by single-pass hot compression tests and correspondingly constitutive equations were developed in terms of flow stress-strain curve. Furthermore, processing maps were derived to analyze the relationship between microstructural evolution and deformation conditions in conjunction with the experimental microstructural observations. The purpose of this study is to analyze the hot deformation behavior of medium-Mn steel, and to predict the optimal hot processing parameters.

\section{Experimental}

The experimental steel was supplied by Ansteel, in the form of a billet with dimensions $80 \mathrm{~mm} \times 80 \mathrm{~mm} \times 80$ $\mathrm{mm}$. The chemical composition of the steel is given in Table 1. The specimen used in this work was cut from a 20-mm-thick plate and subjected to hot rolling at a reduction of about $75 \%$. Cylindrical specimens with diameter of $8 \mathrm{~mm}$ and height $15 \mathrm{~mm}$ were machined from the as-received plate with the compression axis parallel to the normal direction (ND). Thermodynamic analysis of the steel was carried out using Thermo-Calc.

Table 1 Chemical compositions of experimental steel (wt\%)

\begin{tabular}{llllll}
\hline $\mathrm{Mn}$ & $\mathrm{Si}$ & $\mathrm{C}$ & $\mathrm{P}$ & $\mathrm{S}$ & $\mathrm{Fe}$ \\
\hline 8.94 & 2.99 & 0.22 & 0.004 & 0.004 & Bal. \\
\hline
\end{tabular}

Hot compression tests were performed using MMS-300 thermo-mechanical simulator at five different temperatures $\left(850{ }^{\circ} \mathrm{C}, 900{ }^{\circ} \mathrm{C}, 950{ }^{\circ} \mathrm{C}, 1000{ }^{\circ} \mathrm{C}\right.$ and $\left.1050{ }^{\circ} \mathrm{C}\right)$ and four different strain rates $\left(0.05 \mathrm{~s}^{-1}, 1 \mathrm{~s}^{-1}, 5 \mathrm{~s}^{-1}\right.$ and $\left.10 \mathrm{~s}^{-1}\right)$. The details of hot compression tests are illustrated in Fig. 1. Specimens were reheated to $1200{ }^{\circ} \mathrm{C}$ at the rate of $10^{\circ} \mathrm{C} / \mathrm{s}$, held for $180 \mathrm{~s}$ in order to homogenize microstructure, then cooled at the rate of $5^{\circ} \mathrm{C} / \mathrm{s}$ to deformation temperature and held for $10 \mathrm{~s}$ to eliminate thermal gradient prior to the compression tests. After compression, all the specimens were immediately quenched to retain the deformed microstructure at room temperature. The true strain in this work is 0.6. To observe initial microstructure before compression, sample was heated to $1200{ }^{\circ} \mathrm{C}$ at the rate of $10{ }^{\circ} \mathrm{C} / \mathrm{s}$ and held for $180 \mathrm{~s}$ followed by water quenching. A commercial software, Image-Pro Plus, was used to measure the average grain size. The result is shown in Fig. 2. The microstructure consisted of fully recrystallized grains with average size of $\sim 50 \mu \mathrm{m}$.

Samples for microstructural examination were cut parallel to the compression axis, followed by mechanical polishing and etching in a solution of saturated picric acid with $0.2 \%$ hydrofluoric acid to reveal the prior austenite boundary. Microstructure examination was conducted using a Zeiss sigma 300.

\section{Results and Discussion}

\subsection{Thermodynamic Calculation}

Figure 3 illustrates the equilibrium phase diagram at different carbon contents and equilibrium phase constituents for the experimental steel. As seen in Fig. 3a (red broken

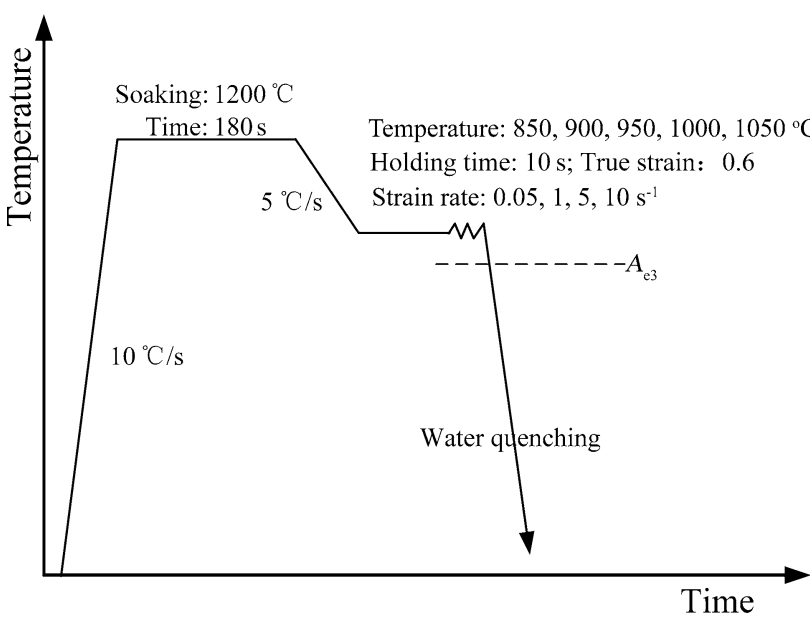

Fig. 1 Time-temperature detail of hot compression tests for experimental steel $\left(A_{\mathrm{e} 3}\right.$ : equilibrium transformation temperature from austenite to ferrite) 


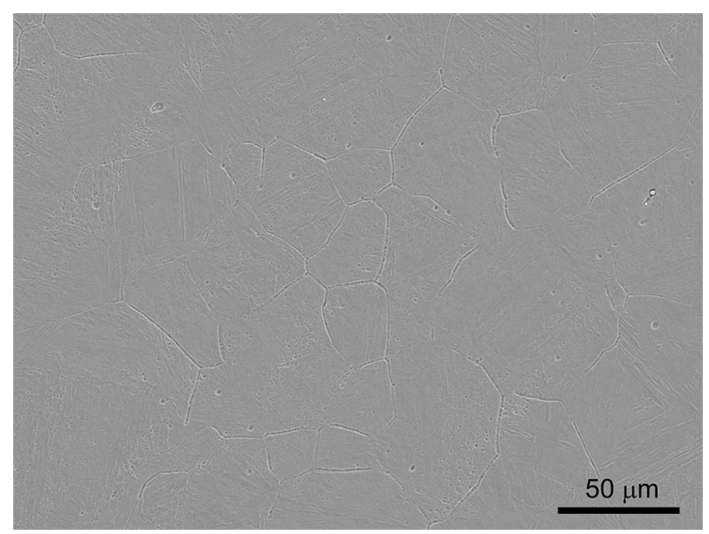

Fig. 2 Initial microstructure of steel before compression

line), the equilibrium phase of studied steel consists of ferrite, retained austenite and cementite at room temperature, and the temperature of liquid phase is $\sim 1450{ }^{\circ} \mathrm{C}$. The temperature of austenite transformation is $\sim 620^{\circ} \mathrm{C}$, and it ends at $680{ }^{\circ} \mathrm{C}$. According to Fig. $3 \mathrm{~b}$, the fraction of austenite is $\sim 99.9 \%$ above $680{ }^{\circ} \mathrm{C}$. The fraction of ferrite with BCC structure is close to $50 \%$ at $597{ }^{\circ} \mathrm{C}$. Below $623{ }^{\circ} \mathrm{C}$, carbides start to precipitate and form cementite. In general, the temperature of hot deformation in an industrial setting is between 700 and $1200{ }^{\circ} \mathrm{C}$, and in this temperature range, the steel is in austenite single-phase region. The austenite is inclined to occurrence of DRX during hot deformation due to low stacking fault energy, which has significant influence on flow stress and microstructural evolution.

\subsection{Flow Stress Behavior}

Typical stress versus strain curves of steel at different deformation temperatures $\left(850{ }^{\circ} \mathrm{C}, \quad 900{ }^{\circ} \mathrm{C}, 950{ }^{\circ} \mathrm{C}\right.$, $\left.1000{ }^{\circ} \mathrm{C}, 1050{ }^{\circ} \mathrm{C}\right)$ and various strain rates $\left(10 \mathrm{~s}^{-1}, 5 \mathrm{~s}^{-1}\right.$, $1 \mathrm{~s}^{-1}, 0.05 \mathrm{~s}^{-1}$ ) are illustrated in Fig. 4. As expected, the flow stress is strongly dependent on the deformation temperature and strain rate. All the curves show a similar behavior where the flow stress increases with decreasing deformation temperature and increasing strain rate. The main reason for this behavior is that higher deformation temperature weakens resistance to dislocation motion, thereby reducing work hardening (WH) [27].

According to Fig. 4a, the initial WH increases the flow stress to a maximum at small strain, and then, the growth rate continuously decreases until the flow stress reaches a steady state. This is widely recognized to be a consequence of dynamic recovery (DRV) process. The flow curves obtained at higher temperature and lower strain rates $\left(0.05 \mathrm{~s}^{-1}\right.$ and $900-1050{ }^{\circ} \mathrm{C}, 1 \mathrm{~s}^{-1}$ and $1000-1050{ }^{\circ} \mathrm{C}$, shown as Fig. 4b-e) exhibit a typical DRX behavior. After initial deformation-strengthened stage caused by $\mathrm{WH}$, the nucleation and growth of new DRX grains release the energy of distortion and absorb the defects, such as dislocations and sub-boundaries formed during deformation, as well as producing softening. As a result, the flow stress decreases with the increase in strain. Moreover, unlike other deformation temperature, all the flow curves deformed at $1050{ }^{\circ} \mathrm{C}$ exhibit a significant DRX behavior even at the strain rates of $10 \mathrm{~s}^{-1}$. The mobility of grain boundary and dislocation are higher at high temperature, which is beneficial for the occurrence of DRX [28, 29].
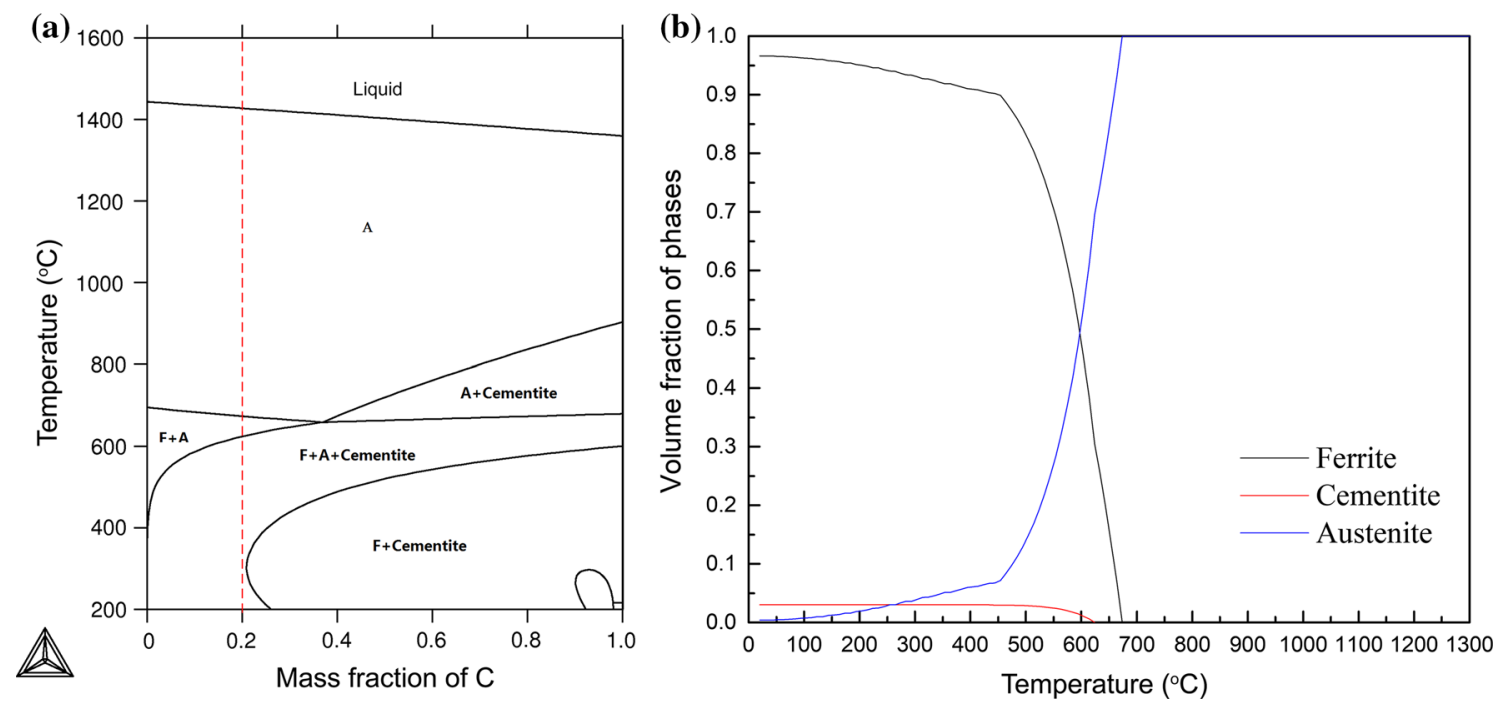

Fig. 3 Equilibrium phase diagrams a, equilibrium phase constituents $\mathbf{b}$ predicted by Thermo-Calc for tested steel 

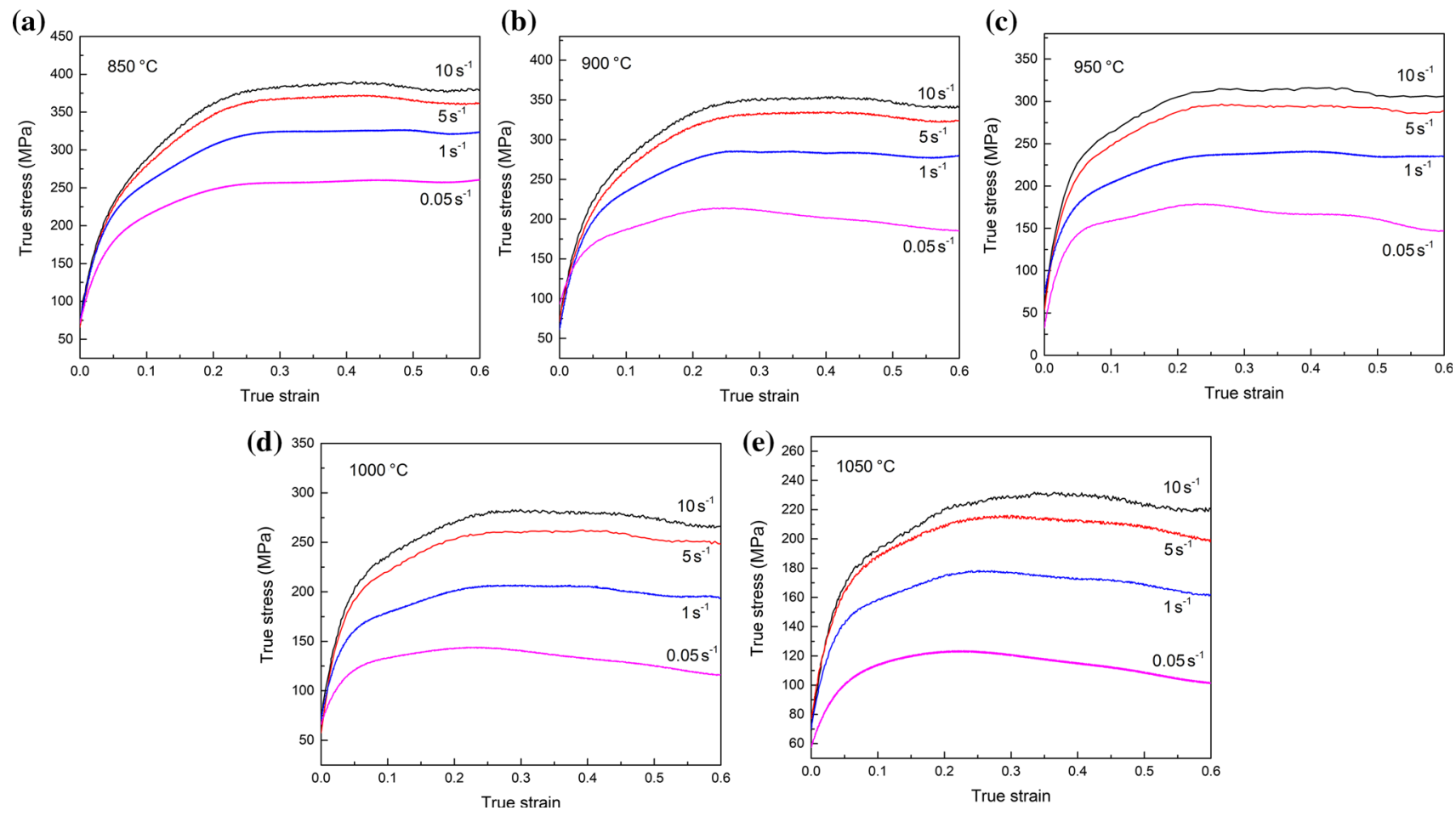

Fig. 4 Flow stress-strain curves of tested steel at different strain rates and temperatures: a $850{ }^{\circ} \mathrm{C} ; \mathbf{b} 900{ }^{\circ} \mathrm{C} ; \mathbf{c} 950{ }^{\circ} \mathrm{C} ; \mathbf{d} 1000{ }^{\circ} \mathrm{C}$; e $1050{ }^{\circ} \mathrm{C}$

\subsection{Constitutive Modeling Flow Curves}

Constitutive equations are generally used to calculate and analyze the activation and the relationship among deformation temperature, flow stress and strain. Zener-Hollomon equation can characterize the effect of deformation temperature and strain rate, especially at high-deformation temperature, on the deformation process [30-32]. The corresponding equations are as followed:

$\dot{\varepsilon}=A f(\sigma) \exp \left(-\frac{Q}{R T}\right)$,

$Z=\dot{\varepsilon} \exp \left(\frac{Q}{R T}\right)$

where $\dot{\varepsilon}$ is the strain rate $\left(\mathrm{s}^{-1}\right), Q$ is the activation energy $(\mathrm{J} / \mathrm{mol}), \sigma$ is the flow stress (MPa), $T$ is the absolute temperature $(\mathrm{K}), A$ is the material constant, $R$ is the universal constant $(8.341 \mathrm{~J} / \mathrm{mol}), f(\sigma)$ is a function of stress and $Z$ is the Zener-Hollomon parameter. As seen in Eqs. (1) and (2), the Zener-Hollomon parameter $(Z)$ is considered as a function of flow stress and can be divided into three equations suitable for different stress levels (power function, low stress; exponential function, high stress; and hyperbolic sine function, any stress): $f(\sigma)=\left\{\begin{array}{lc}\sigma^{n_{1}} & \alpha \sigma<0.8 \\ \exp (\beta \sigma) & \alpha \sigma>1.2, \\ {[\sinh (\alpha \sigma)]^{n}} & \text { for all } \sigma\end{array}\right.$

where $n_{1}, n, \alpha$ and $\beta$ are material constants, $\alpha=\beta / n_{1}$. Substituting Eq. (3) into Eq. (1) and taking the natural logarithms of both sides of the equation yields:

$\ln \alpha=\frac{1}{n_{1}} \ln \dot{\varepsilon}-\frac{1}{n_{1}} \ln B \quad$ (low stress level),

$\sigma=\frac{1}{\beta} \ln \dot{\varepsilon}-\frac{1}{\beta} \ln C \quad$ (high stress level),

$\ln [\sinh (\alpha \sigma)]=\frac{1}{n} \ln \dot{\varepsilon}+\frac{Q}{n R}\left(\frac{1}{T}\right)-\frac{1}{n} \ln A$

(any stress level),

where $B$ and $C$ are material constants and are independent of deformation temperature. In this study, taking strain $\varepsilon=0.2$ as an example, the relationship between $\ln \sigma-\ln \dot{\varepsilon}$ and $\sigma-\ln \dot{\varepsilon}$ are shown in Fig. 5. The plots in Fig. 5 are essentially linear $\left(R^{2}>0.95\right.$, correlation coefficient $)$ and parallel. The values of $n_{1}$ and $\beta$ can be determined from the slope of the linear regression lines. Thus, based on $\alpha=\beta$ / $n_{1}$, the values of material constants can be calculated.

When strain rate is constant, the Eq. (6) can be expressed by: 
(a)

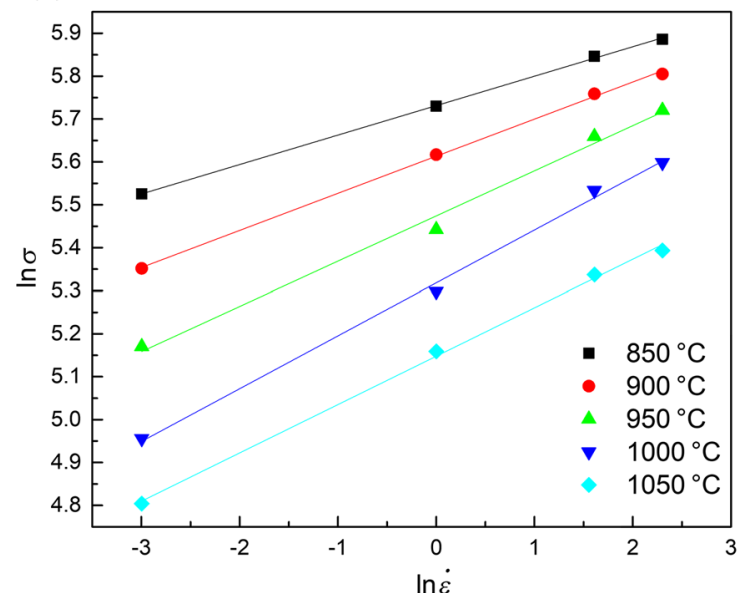

(b)

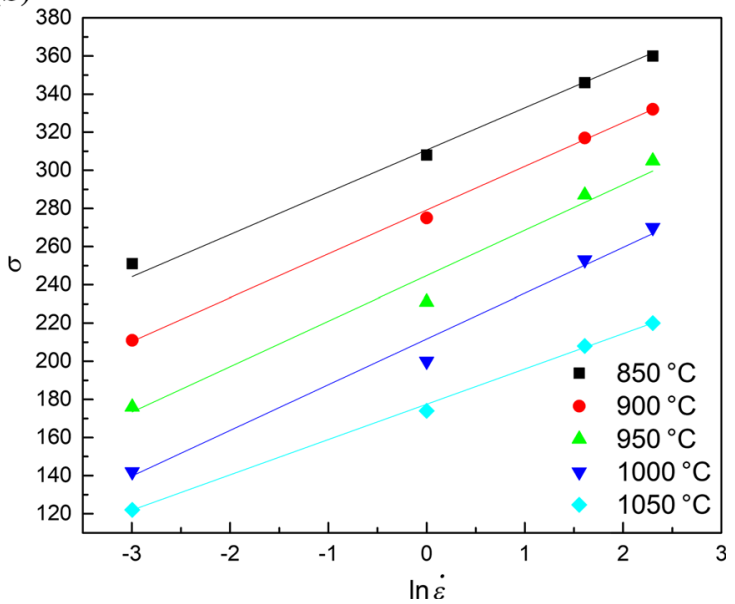

Fig. 5 Relationship between $\ln \sigma-\ln \dot{\varepsilon} \mathbf{a}, \sigma-\ln \dot{\varepsilon} \mathbf{b}$ at $\varepsilon=0.2$

$\ln [\sinh (\alpha \sigma)]=\frac{Q}{10,000 \cdot(n R)} \frac{10,000}{T}+\frac{\ln \dot{\varepsilon}-\ln A}{n}$.

The relationship between $\ln [\sinh (\alpha \sigma)]-\ln \quad \dot{\varepsilon}$ and $\ln [\sinh (\alpha \sigma)]-10,000 / T$ at different temperatures is obtained, in terms of Eqs. (6) and (7), as shown in Fig. 6. Similarly, the value of material constant $\mathrm{n}$ can be calculated from the slope of the lines in Fig. 6. Thus, the slope of the linear regression lines in Fig. $6 \mathrm{~b}$ gives the value of $Q /(10,000 n R)$, and the activation energy of the experimental steel is obtained.

In previous studies, flow stress equation was used to describe the flow behavior during hot deformation by Arrhenius equations [33-35]. However, Lin and Chen [17] reviewed the constitutive equations and found that the flow stress equations in Refs. [32, 33] did not consider the effect of strain. Therefore, they established a modified model that compensated for strain and strain rate and considered $Q, n$, $A$ and $\alpha$ as a polynomial function of strain [36].

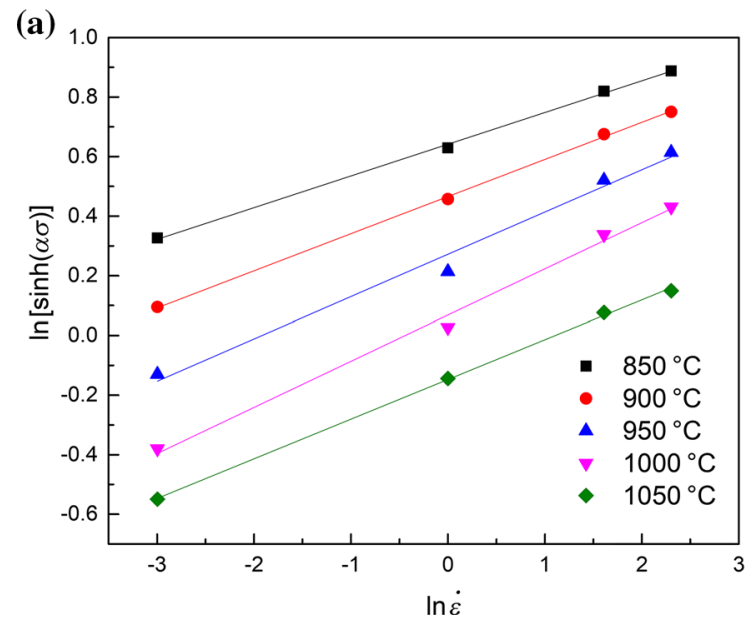

In our study, we modified constitutive models and calculated the material constants of investigated steel $(Q, n, A$ and $\alpha$ ) at different strain (strains $0.1-0.6$ ) because of a complex relationship between material constants and strain. Figure 7 illustrates the relationships of material constants for the experimental steel. The relationship functions can be obtained by fitting with a 6th order polynomial. Thus, the polynomial functions can be written as:

$\left\{\begin{array}{l}\alpha=a_{0}+a_{1} \varepsilon+a_{2} \varepsilon^{2}+a_{3} \varepsilon^{3}+a_{4} \varepsilon^{4}+a_{5} \varepsilon^{5}+a_{6} \varepsilon^{6} \\ n=b_{0}+b_{1} \varepsilon+b_{2} \varepsilon^{2}+b_{3} \varepsilon^{3}+b_{4} \varepsilon^{4}+b_{5} \varepsilon^{5}+b_{6} \varepsilon^{6} \\ Q=c_{0}+c_{1} \varepsilon+c_{2} \varepsilon^{2}+c_{3} \varepsilon^{3}+c_{4} \varepsilon^{4}+c_{5} \varepsilon^{5}+c_{6} \varepsilon^{6} \\ \ln A=d_{0}+d_{1} \varepsilon+d_{2} \varepsilon^{2}+d_{3} \varepsilon^{3}+d_{4} \varepsilon^{4}+d_{5} \varepsilon^{5}+d_{6} \varepsilon^{6}\end{array}\right.$.

The corresponding coefficients of polynomial functions are summarized in Table 2.

As seen in Fig. 7a, the $\alpha$ value decreased with increasing strain, then reached its minimum value $\left(0.0044 \mathrm{MPa}^{-1}\right)$ at

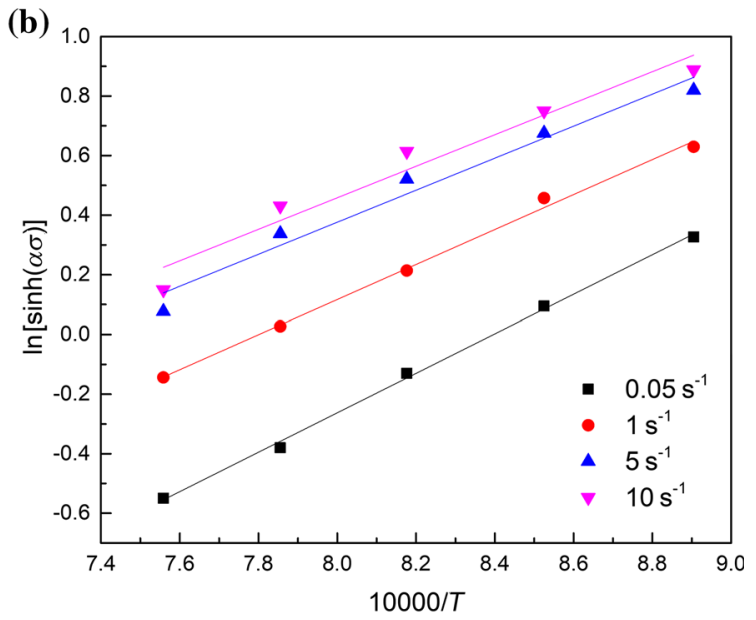

Fig. 6 Relationship between $\ln [\sinh (\alpha \sigma)]-\ln \dot{\varepsilon} \mathbf{a}, \ln [\sinh (\alpha \sigma)]-10,000 / T \mathbf{b}$ 
(a)

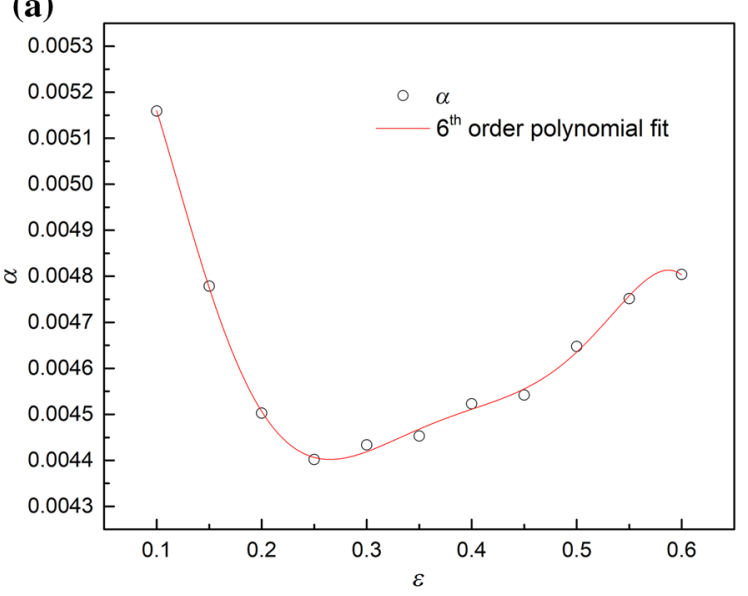

(c)

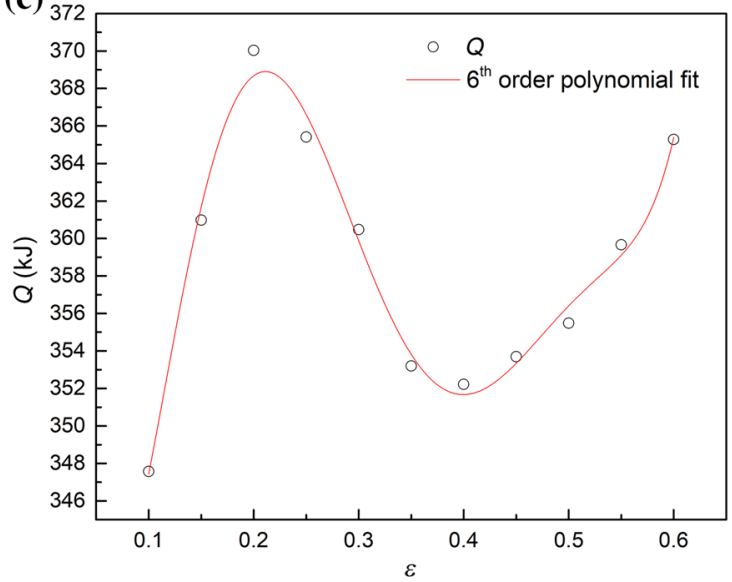

(b)

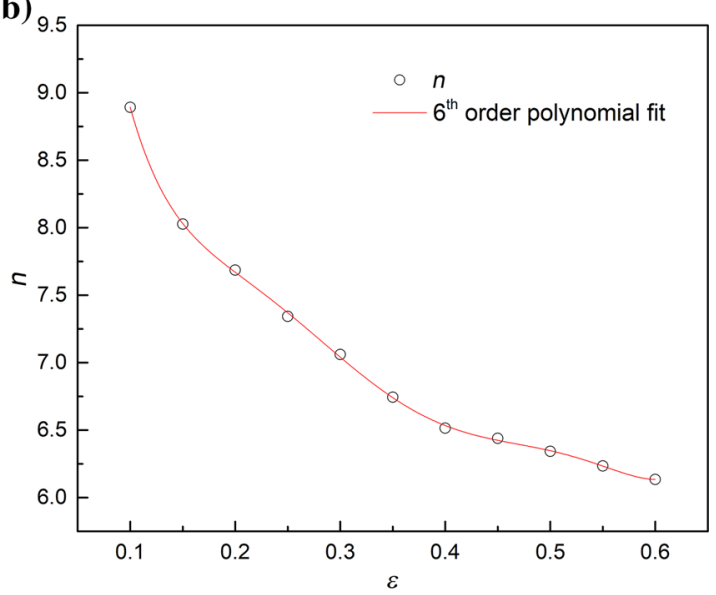

(d)

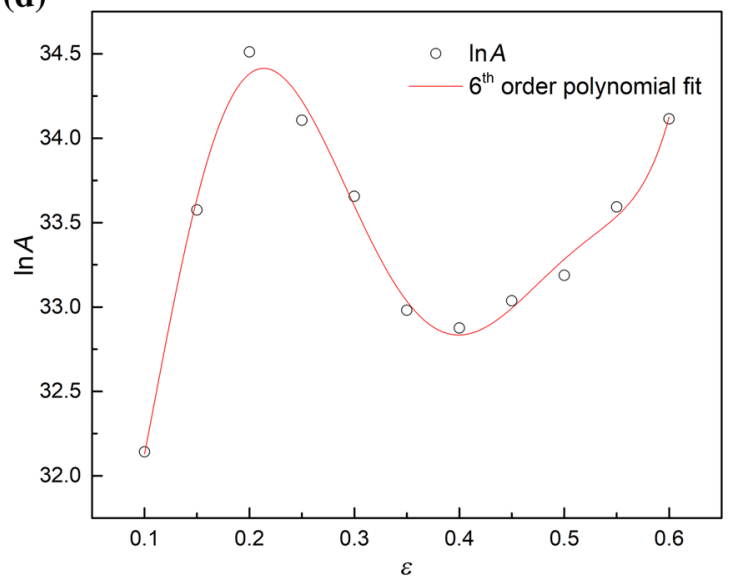

Fig. 7 Relationship among $\alpha \mathbf{a}, n \mathbf{b}, Q \mathbf{c}, \ln A \mathbf{d}$ with true strain

Table 2 Coefficients of polynomial functions

\begin{tabular}{llllllll}
\hline & $i=0$ & $i=1$ & $i=2$ & $i=3$ & $i=4$ & $i=5$ & $i=6$ \\
\hline$a_{i}$ & 0.00472 & 0.02897 & -0.41598 & 2.18992 & -5.53854 & 6.81792 & -3.27803 \\
$b_{i}$ & 17.28371 & -174.879 & 1354.541 & -5528.39 & $12,060.81$ & $-13,328.9$ & 5865.289 \\
$c_{i}$ & 34.38965 & -142.208 & 2075.295 & $-11,380.1$ & $29,302.64$ & $-35,982.9$ & $17,070.85$ \\
$d_{i}$ & $38,1403.3$ & $-1.68 \mathrm{E}+6$ & $2.29 \mathrm{E}+7$ & $-1.23 \mathrm{E}+8$ & $3.15 \mathrm{E}+8$ & $-3.86 \mathrm{E}+8$ & $1.83 \mathrm{E}+8$ \\
\hline
\end{tabular}

the strain of 0.25 and finally increased up to $0.0048 \mathrm{MPa}^{-1}$. It has been suggested that the $\alpha$ value is altered for different materials and deformation conditions [37]. The value of $n$ decreased steadily with increasing strain, lying in the range of 6.134-8.890. It reflected that better workability occurred at the large strain level. Referring to Fig. 7c, the value of activation energy $Q$ changed in the range of $347.5-370.0 \mathrm{~kJ} / \mathrm{mol}$, with a mean value of $\sim 358.5 \mathrm{~kJ} / \mathrm{mol}$. Compared to the reported values for hot deformation of high-Mn steels such as $387.84 \mathrm{~kJ} / \mathrm{mol}$ in Fe-20Mn-3Si-3Al [38] and $439 \mathrm{~kJ} / \mathrm{mol}$ in $\mathrm{Fe}-23 \mathrm{Mn}-0.6 \mathrm{C}$ [39], the obtained values are significantly lower. This difference in activation energy has been suggested to relate to the high solute content present in
high-Mn steel [40]. The obtained values of $Q$ are close to those reported austenitic stainless steels with range of $350-510 \mathrm{~kJ} / \mathrm{mol}$ [37].

Finally, the material constants were evaluated and the $Z$ parameter can be expressed in terms of stress and strain. The flow stress can be predicted as a function of ZenerHollomon parameter, which involves the effect of strain rate and deformation temperature, and material constants introduced the effect of strain, given by:

$\sigma=\frac{1}{\alpha} \ln \left\{\left(\frac{Z}{A}\right)^{1 / n}+\left[\left(\frac{Z}{A}\right)^{2 / n}+1\right]^{1 / 2}\right\}$. 


\subsection{Verification of Constitutive Equations}

The flow stress equation, Eq. (9), takes the effects of deformation temperature, strain rate and strain on the flow stress behavior into consideration; thus, errors from diverse elements are unavoidable. We compared the calculated data from the established flow stress equation with experimental data. As shown in Fig. 8, the calculated flow stresses fit well with the experimental data throughout the entire strain rate range except some noticeable deviations, for example, $850{ }^{\circ} \mathrm{C}$ and $10 \mathrm{~s}^{-1}, 850{ }^{\circ} \mathrm{C}$ and $0.05 \mathrm{~s}^{-1}$ and $1050{ }^{\circ} \mathrm{C}$ and $5 \mathrm{~s}^{-1}$. Thus, we can study the flow softening behavior using the established flow stress equation.

To further assess the reliability of the established flow stress equations, we introduced the correlation coefficient $(R)$ and the average absolute relative error (AARE), which can be expressed by:

$R=\frac{\sum_{i=1}^{N}\left(E_{i}-\bar{E}\right)\left(C_{i}-\bar{C}\right)}{\sqrt{\sum_{i=1}^{N}\left(E_{i}-\bar{E}\right)^{2} \sum_{i=1}^{N}\left(C_{i}-\bar{C}\right)^{2}}}$,

$\operatorname{AARE}(\%)=\frac{1}{N} \sum_{i=1}^{N}\left|\frac{E_{i}-C_{i}}{E_{i}}\right| \times 100$,

where $E$ is the experimental data, $C$ is the calculated data by the developed equations. $\bar{E}$ and $\bar{C}$ are the mean value of
$E$ and $C$, respectively. $N$ is the number of experimental data. The correlation coefficient $(R)$ is usually used to analyze the linearity between experimental and calculated data, and AARE reflects the average-relative error. Therefore, combining $R$ with AARE can precisely assess the calculated data.

Figure 9 shows a good correlation between the experimental and calculated data. The value of $R$ is 0.988 . It indicates that the predicted curves can basically reflect the shape of experimental flow stress. As shown in Fig. 10, it can be seen that the maximum deviation occurred at $1000{ }^{\circ} \mathrm{C}$ at the strain rate of $0.05 \mathrm{~s}^{-1}$, with the AARE being $6.21 \%$. The errors shown in Fig. 10 can be accounted of three factors: (1) the experimental error in measuring flow stress; (2) the error from fitting to obtain the material constants; (3) the error from the model itself used in this work. Beside the deviation, the AARE for the entire deformation condition range investigated is $2.74 \%$. Therefore, it can be considered that the established constitutive equation can adequately predict the flow stress in terms of AARE and $R$.

\subsection{Processing Maps}

Processing and instability maps are widely used to study the constitutive behavior and optimize the hot deformation processes. According to the DMM, the hot deformation process can be considered as a nonlinear energy dissipater (a)

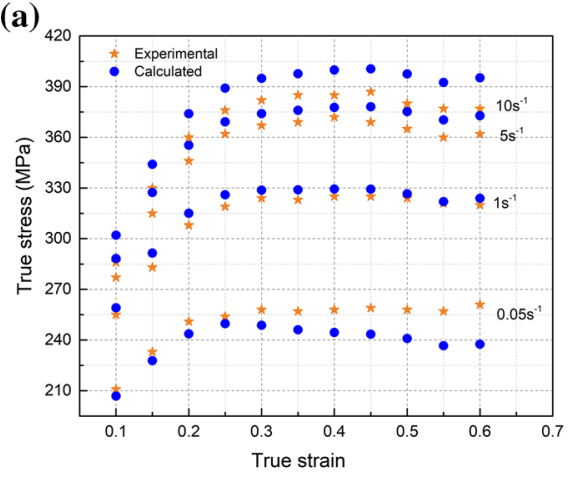

(d)

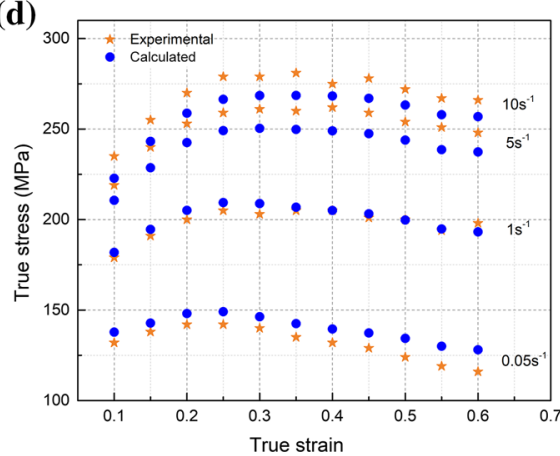

(c)

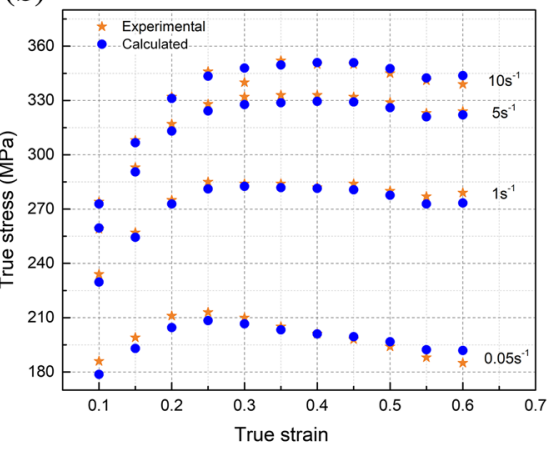

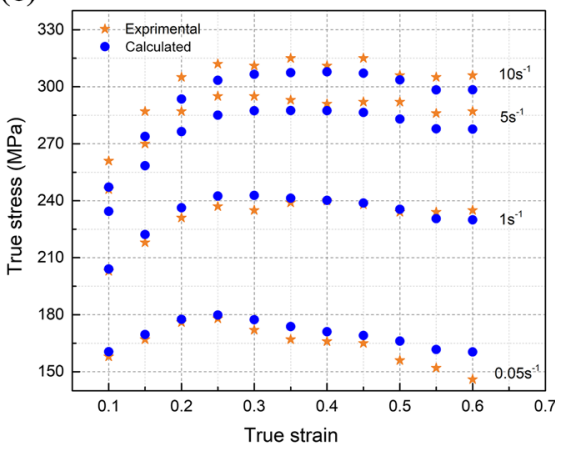

(e)

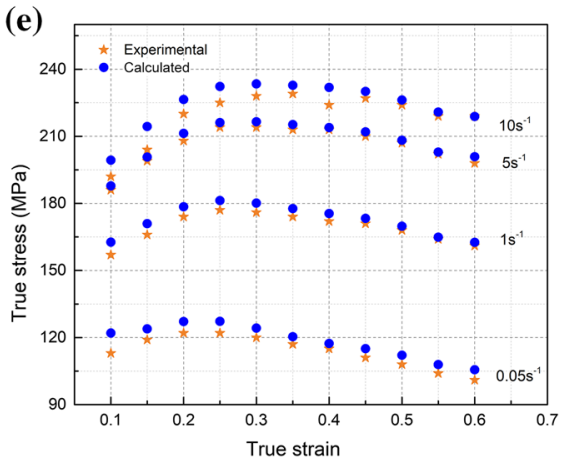

Fig. 8 Comparison of experimental and calculated data at deformation temperatures of $850{ }^{\circ} \mathrm{C} \mathbf{a}, 900{ }^{\circ} \mathrm{C} \mathrm{b}, 950{ }^{\circ} \mathrm{C} \mathrm{c}, 1000{ }^{\circ} \mathrm{C} \mathrm{d}, 1050{ }^{\circ} \mathrm{C} \mathbf{e}$ 


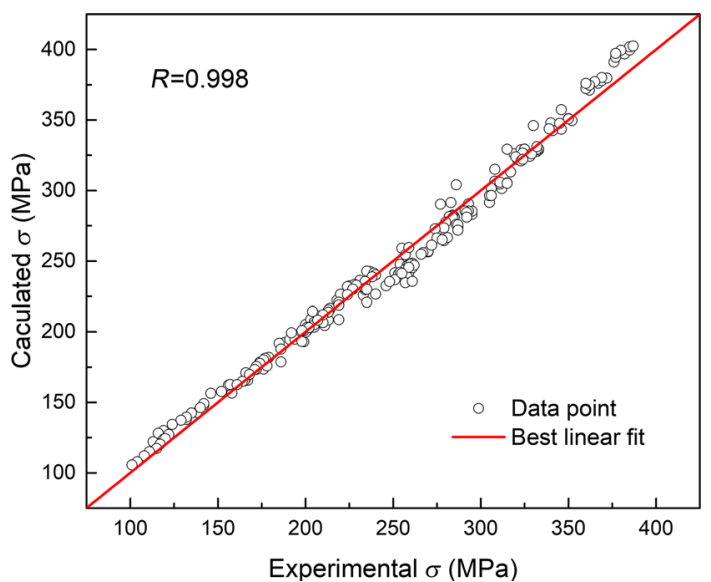

Fig. 9 Correlation between experimental and calculated data from established equations

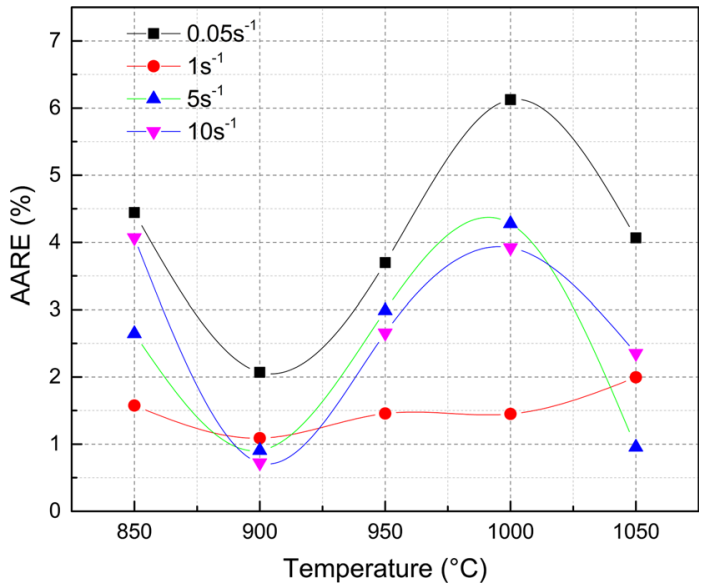

Fig. 10 Average absolute relative error (AARE) at different deformation conditions

system, which contains the power dissipated by deformation $(G)$ and the power dissipated by microstructural changes $(J)$. Therefore, the energy input in the workpiece $(P=\sigma \dot{\varepsilon})$ can be expressed as:

$P=G+J=\int_{0}^{\sigma} \dot{\varepsilon} \mathrm{d} \sigma+\int_{0}^{\dot{\varepsilon}} \sigma \mathrm{d} \dot{\varepsilon}$.

In this model, the value of $G / J$ is defined as the strain rate sensitivity $(m)$. The parameter, $m$, can be expressed as:

$m=\left(\frac{\partial J}{\partial G}\right)_{\varepsilon, T}=\frac{\partial P}{\partial G} \frac{\partial J}{\partial P}=\frac{\sigma d \dot{\varepsilon}}{\dot{\varepsilon} d \sigma}=\left[\frac{\partial \ln \sigma}{\partial \ln \dot{\varepsilon}}\right]_{\varepsilon, T}$.

For an ideal linear dissipater process (when $m=1$ ), $J$ reaches the maximum value $\left(J_{\max }=\sigma \dot{\varepsilon} / 2=P / 2\right)$. The efficiency of energy dissipation, $\eta$, which describes the relationship between microstructural changes and energy dissipation, is defined as:
$\eta_{\mathrm{DMM}}=\frac{J}{J_{\max }}=\frac{2 m}{m+1}$.

According to Eqs. (13) and (14), the efficiency of energy dissipation is a function of $T$ and $\dot{\varepsilon}$. The variation of $\eta$ with $T$ and $\dot{\varepsilon}$, under the condition of constant $\varepsilon$, constitutes the power dissipation map, in which different regions represent different microstructures. The high value of $\eta$ almost relates to excellent plasticity, resulting in stable microstructural change. However, the value of $\eta$ in the instability region sometimes may be higher. Thus, it needs additional criteria to analyze the workability for hot processing.

The instability criteria used in this study is the Prasad criteria based on DMM and Ziegler's plastic flow theory. Prasad criteria for predicting the flow instability during hot compression is:

$\xi_{P}(\dot{\varepsilon}, T)=\frac{\partial \ln \left(\frac{m}{m+1}\right)}{\partial \ln \dot{\varepsilon}}+m<0$.

Figure 11 presents the processing maps of the experimental steel, including power dissipation maps (iso-efficiency contours) and instability maps (marked as red area, the value of $\xi_{\mathrm{p}}(\dot{\varepsilon})$ is negative). The distributions of counter lines are similar at true strains of 0.20 .3 and 0.4 , and the peak value of energy dissipation is emerged at the deformation temperature of $940-1030{ }^{\circ} \mathrm{C}$. With the strain increasing from 0.4 to 0.6 , the maximum energy dissipation $(\eta)$ increases and appears to move slightly to the region of higher temperature and lower strain rate. According to Refs. [31, 32], the high $\eta$ is corresponding to the occurrence of dynamic recrystallization (DRX). In these regions, the flow stress of material decreases with true strain and the material undergoes flow softening. Therefore, it infers that the DRX is likely to be occurred at this region for experimental steel and reaching maximum at peak $\eta$. Additionally, a wide instability region could be found in the upper part of the processing maps, corresponding to the regions of low temperatures and high strain rate. The information from the processing maps could be applied to industrial working process for experimental steel. The temperature and strain rate failing in region of high $\eta$ are supposed to be the optimum working area; simultaneously the working parameters for avoiding flow instability can be obtained from the processing map (red regions in Fig. 11).

Figure 12 exhibits the original austenite grain boundaries of the specimen after compression. It can also be seen that DRX occurs when deformed at $1050{ }^{\circ} \mathrm{C}$, and the average grain size decreased (from 16 to $8 \mu \mathrm{m}$ ) with increasing strain rate from 0.05 to $10 \mathrm{~s}^{-1}$. This further confirms the discussion above, namely deformation at high $\eta$ region is corresponding to DRX resulting in fine recrystallized grains. Moreover, the representative 


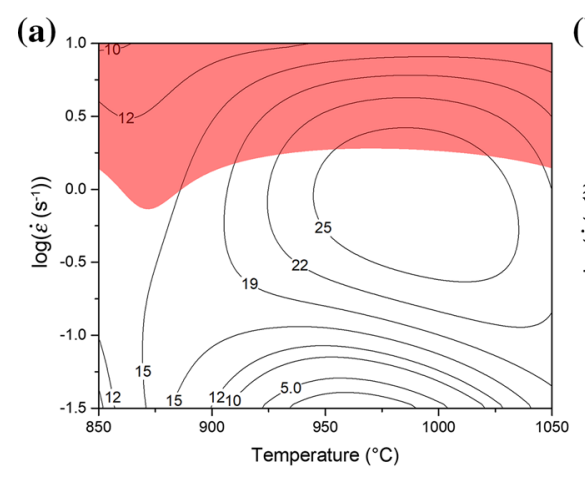

(d)

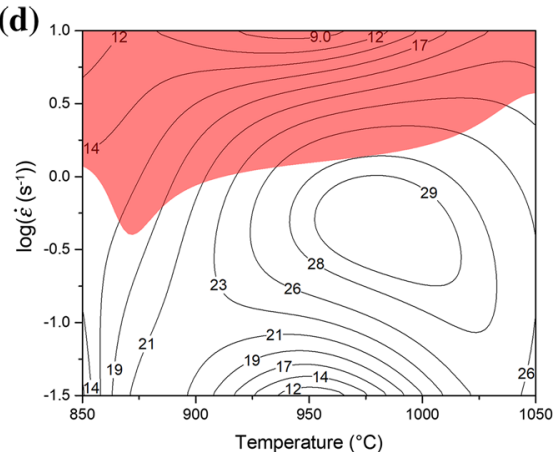

(b)

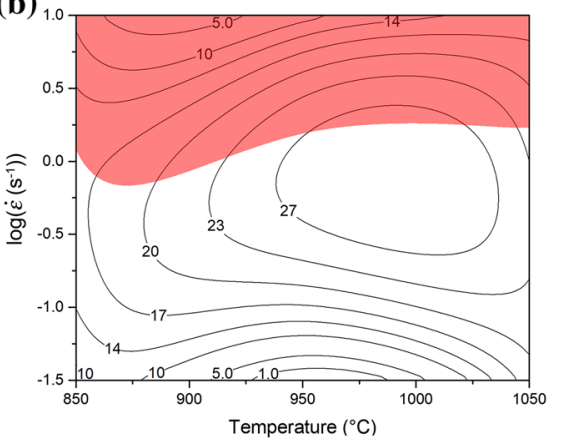

(c)

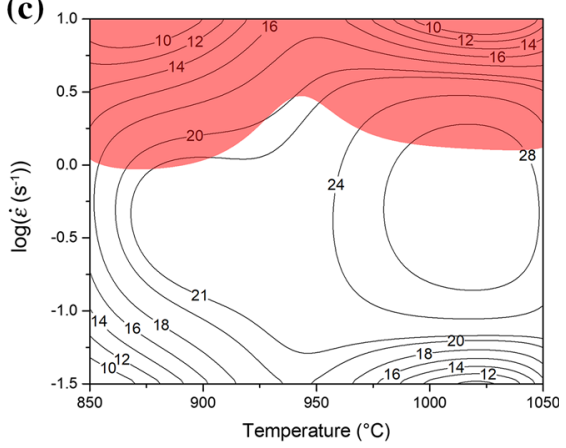

(e)

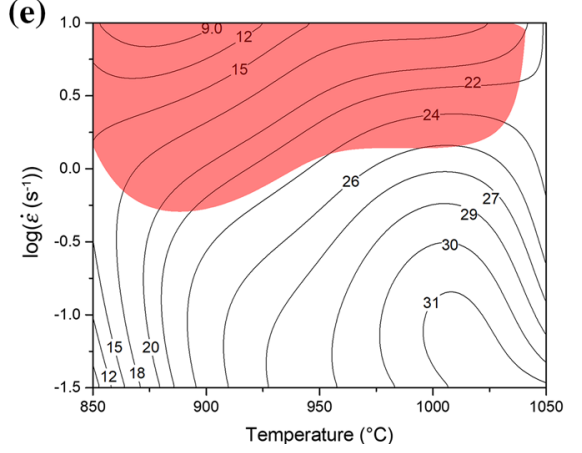

Fig. 11 Processing maps for alloyed steel at true strains of $0.2 \mathbf{a}, 0.3 \mathbf{b}, 0.4 \mathbf{c}, 0.5 \mathbf{d}, 0.6 \mathbf{e}$
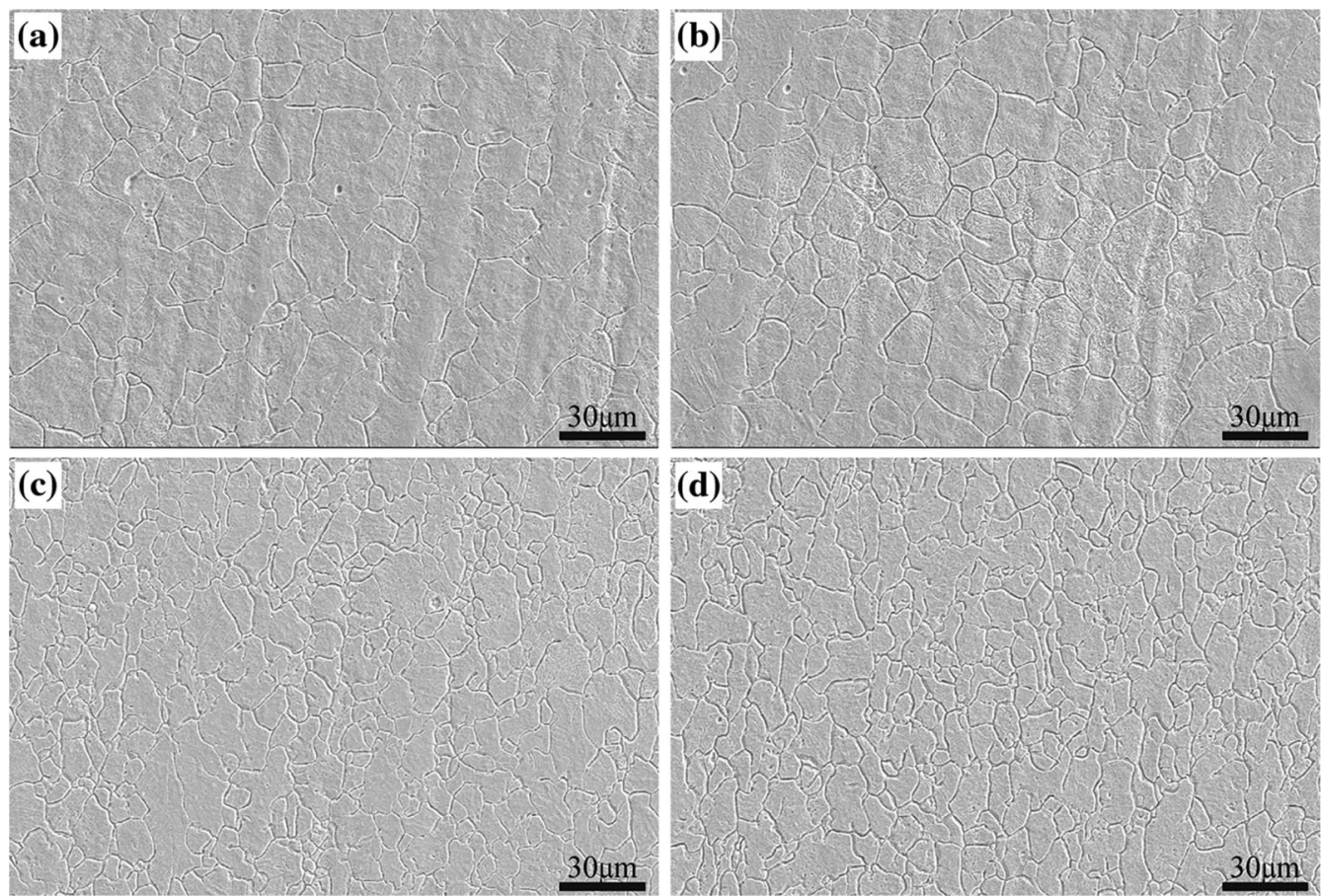

Fig. 12 SEM images of compressed samples deformed at $1050{ }^{\circ} \mathrm{C}$ with strain rates of $0.05 \mathrm{~s}^{-1} \mathbf{a}, 1 \mathrm{~s}^{-1} \mathbf{b}, 5 \mathrm{~s}^{-1} \mathbf{c}, 10 \mathrm{~s}^{-1} \mathbf{d}$

microstructures of the specimens deformed in the instability region are observed, as shown in Fig. 13. Typically, the flow instability mechanisms are associated with flow localization, adiabatic shear bands or deforming cracks. For experimental steel, only flow localization is occurred, resulting in elongated original grains and curved grain boundaries along deformation direction, as indicated in red arrows in Fig. 13. Additionally, partially recrystallized grains are observed due to severe flow localization, as indicated in blue arrows in Fig. 13. The inhomogeneous 


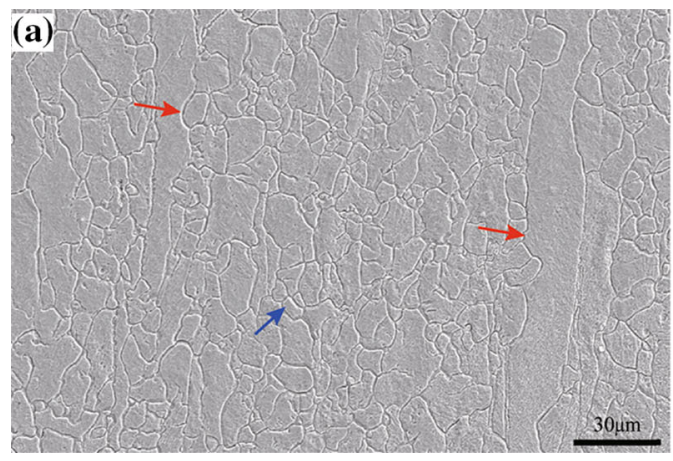

Fig. 13 SEM images of specimens deformed in instability region: a 900

microstructure presented in Fig. 13 indicates that the flow instability regions should be avoided for hot processing.

In summary, the optimal deformation condition of the experimental steel is $1050{ }^{\circ} \mathrm{C}, 0.05-10 \mathrm{~s}^{-1}$, which can be applied to industrial working process. Under these conditions, a significant amount of fully recrystallized structure can be obtained. For designing and industrial manufacture, high strain rate and low temperature should be prohibited due to the flow instability, as well as inhomogeneous microstructure.

\section{Conclusions}

1. The equilibrium phase diagram at different $\mathrm{C}$ contents and equilibrium phase constituents were calculated by Thermo-Calc. The fraction of austenite was $99.9 \%$ above $680{ }^{\circ} \mathrm{C}$ and carbides in austenite start to dissolve and transform to cementite below $623{ }^{\circ} \mathrm{C}$. The best hot working temperature for the steel was greater than $680{ }^{\circ} \mathrm{C}$, where the microstructure consists of austensite phase.

2. The studied steel exhibited conspicuous flow softening behavior at higher deformation temperature range of $950-1050{ }^{\circ} \mathrm{C}$ and lower strain rate range of $0.05-5 \mathrm{~s}^{-1}$. This behavior increased with the increasing temperature and decreasing strain rate. The flow softening behavior was caused by deformation heating, DRX and flow instability.

3. Constitutive equations were developed based on the modified-Arrhenius equation which are widely used to describe the relationship among strain rate, flow stress and deformation temperature. The established equations as function of strain included the effects of stain rate, deformation and strain. The results indicated that these constitutive models accurately describe the flow behavior of the steel.

4. The processing maps were established in terms of Prasad criteria at different strains, and the efficiency of

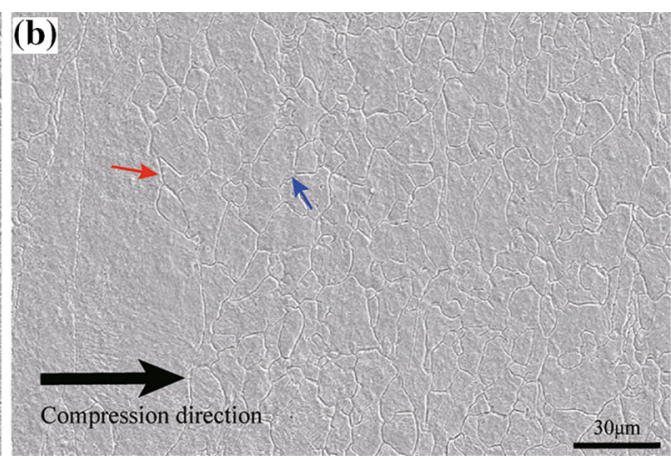

${ }^{\circ} \mathrm{C}, 10 \mathrm{~s}^{-1} ; \mathbf{b} 1000{ }^{\circ} \mathrm{C}, 5 \mathrm{~s}^{-1}$

power dissipation varied with increasing strain. The maps predicted that hot working of experimental steel can be carried out at the condition of $1050{ }^{\circ} \mathrm{C}$, $0.05-10 \mathrm{~s}^{-1}$. The flow localization is mainly flow instability mechanism for experimental steel.

Acknowledgements This work was financially supported by the National Natural Science Foundation of China (No. 51775102) and the National key research and development plan (2017YF0703001). R.D.K. Misra gratefully acknowledges continued collaboration with the Northeastern University as an Honorary Professor by guiding student in research.

\section{References}

[1] M.M. Wang, C.C. Tasan, D. Ponge, A.C. Dippel, D. Raabe, Acta Mater. 85, 216 (2015)

[2] J. Han, S.J. Lee, J.G. Jung, Y.K. Lee, Acta Mater. 78, 369 (2014)

[3] H.W. Luo, J. Shi, C. Wang, W.Q. Cao, X.J. Sun, H. Dong, Acta Mater. 59, 4002 (2011)

[4] J. Hu, L.X. Du, G.S. Sun, H. Xie, R.D.K. Misra, Scr. Mater. 104, 87 (2015)

[5] A. Arlazarov, M. Gouné, O. Bouaziz, A. Hazotte, G. Petitgand, P. Barges, Mater. Sci. Eng. A 542, 31 (2012)

[6] C. Zhao, W.Q. Cao, C. Zhang, Z.G. Yang, H. Dong, Mater. Sci. Technol. 30, 791 (2014)

[7] B.H. Sun, H. Aydin, F. Fazeli, S. Yue, Metall. Mater. Trans. A 47, 1782 (2016)

[8] S.J. Lee, Y.M. Park, Y.K. Lee, Mater. Sci. Eng. A 515, 32 (2009)

[9] J. Han, Y.K. Lee, Acta Mater. 67, 354 (2014)

[10] H. Hong, O.Y. Lee, Acta Mater. 78, 369 (2014)

[11] D.W. Suh, S.J. Park, T.H. Lee, C.S. Oh, S.J. Kim, Metall. Mater. Trans. A 41, 397 (2010)

[12] H.W. Luo, J.H. Liu, H. Dong, Metall. Mater. Trans. A 47, 3119 (2016)

[13] S. Lee, B.C. de Cooman, Metall. Mater. Trans. A 47, 3263 (2016)

[14] Z. Akbari, H. Mirzadeh, J.M. Cabrera, Mater. Des. 77, 126 (2015)

[15] L. Chen, G. Zhao, J. Yu, Mater. Des. 74, 25 (2015)

[16] K. Wu, G. Liu, B. Hu, F. Li, Y. Zhang, Y. Tao, J. Liu, Mater. Charact. 61, 330 (2010)

[17] Y.C. Lin, X.M. Chen, Mater. Des. 32, 1733 (2011)

[18] H.L. Gegel, in Metals Congress, ed. by C.C. Chen (ASM International, Metals Park, 1983), p. 22 
[19] Y.V.R.K. Prasad, S. Sasidhara, Hot Working Guide - A Compendium of Processing Maps (ASM International, 1997)

[20] Y.V.R.K. Prasad, T. Seshacharyulu, Int. Mater. Rev. 43, 243 (1998)

[21] Y.V.R.K. Prasad, T. Seshacharyulu, Mater. Sci. Eng. A 234, 82 (1998)

[22] S.V.S.N. Murty, B.N. Rao, Mater. Sci. Eng. A 254, 76 (1998)

[23] J.Q. Zhang, H.S. Di, H.T. Wang, K. Mao, T.J. Ma, Y. Cao, J. Mater. Sci. 47, 4000 (2012)

[24] P.V. Sivaprasad, S.L. Mannan, Y.V.R.K. Prasad, R.C. Chaturvedi, Mater. Sci. Technol. 17, 545 (2001)

[25] G. Meng, B.L. Li, H.M. Li, Z.R. Nie, Mater. Sci. Eng. A 517, 132 (2009)

[26] P. Cavaliere, E. Cerri, E. Evangelista, Mater. Sci. Eng. A 387-389, 857 (2004)

[27] M.G. Li, X.Y. Yao, J. Luo, Y.Y. Lin, S.B. Sun, H.R. Wang, Acta Metall. Sin. (in Chinese) 43, 937 (2007)

[28] F. Chen, Z.S. Cui, S.J. Chen, Mater. Sci. Eng. A 528, 5073 (2011)

[29] Y.C. Lin, M.S. Chen, J. Zhong, Mech. Res. Commun. 35, 142 (2008)
[30] C. Zener, J.H. Hollomen, Appl. Phys. 15, 22 (1944)

[31] C. Zhang, L.W. Zhang, W.F. Shen, C.R. Liu, Y.N. Xia, R.Q. Li, Mater. Des. 90, 804 (2016)

[32] Y. Zhang, Z. Chai, A.A. Volinsky, B.H. Tian, H.L. Sun, P. Liu, Y. Liu, Mater. Sci. Eng. A 622, 320 (2016)

[33] S. Mandal, V. Rakesh, P.V. Sivaprasad, S. Venugopal, K.V. Kasiviswanathan, Mater. Sci. Eng. A 500, 114 (2009)

[34] D. Samantaray, S. Mandal, A.K. Bhaduri, Mater. Des. 31, 981 (2010)

[35] A. Momeni, K. Dehghani, Mater. Sci. Eng. A 527, 5467 (2010)

[36] Y.C. Lin, M.S. Chen, J. Zhang, J. Compos. Mater. Sci. 424, 470 (2008)

[37] H.J. McQueen, N.D. Ryan, Mater. Sci. Eng. A 322, 43 (2002)

[38] D.J. Li, Y.R. Feng, Z.F. Yin, F.S. Shangguan, K. Wang, Q. Liu, Mater. Des. 34, 713 (2012)

[39] W. Xiong, B. Wietbrock, A. Saeed-Akbari, M. Bambach, G. Hirt, Steel Res. Int. 82, 127 (2011)

[40] D.J. Li, Y.R. Feng, Z.F. Yin, F.S. Shangguan, K. Wang, Q. Liu, Mater. Sci. Eng. A 528, 8084 (2011) 